\title{
Psychiatric Diagnoses of Patients Presenting with Functional Somatic Symptoms in a Primary Care Clinic at a University Teaching Hospital in Nigeria
}

\author{
Chidi J. Okafor ${ }^{1 *}$, Owoidoho Udofia ${ }^{1}$, Essien E. Ekpe ${ }^{2}$ \\ ${ }^{1}$ Department of Psychiatry, University of Calabar, Calabar, Nigeria \\ ${ }^{2}$ Department of Clinical Services, Federal Psychiatric Hospital Calabar, Calabar, Nigeria \\ Email: ${ }^{*}$ dr chidi@yahoo.com
}

Received 8 August 2015; accepted 19 September 2015; published 22 September 2015

Copyright (C) 2015 by authors and Scientific Research Publishing Inc.

This work is licensed under the Creative Commons Attribution International License (CC BY). http://creativecommons.org/licenses/by/4.0/

(c) (i) Open Access

\begin{abstract}
Background: A number of patients that attend primary care medical settings present with distressing bodily complaints which are not attributable to organic (physical) pathology. The difficulties in diagnosing and treating such patients have been observed by many Health Care Practitioners. This study assesses the presence of psychological conditions and attempts to make psychiatric diagnoses (using a standardized diagnostic instrument) in patients presenting with Functional Somatic Symptoms (FSS) in a primary care clinic at the University of Calabar Teaching Hospital. Method: The study was carried out at the General Out Patients Clinic (GOPC) of the University of Calabar Teaching Hospital (UCTH). It was a cross-sectional study of a convenience sample of 100 randomly selected patients presenting with Functional Somatic Symptoms. Those who consented filled the modified version of the Enugu Somatization Scale and were interviewed with the Schedule for Clinical Assessments in Neuropsychiatry (SCAN) for possible psychiatric diagnoses. Results: While 41 of the selected patients were males, 59 were females. Their mean age was 38.5 (SD $=12.2$ ). Frequent headaches and body heat (internal heat) were the most prevalent symptoms in the patients. ICD-10 psychiatry diagnoses using the SCAN were made in $79 \%$ of the patients studied. Depressive disorders (diagnosed in $48 \%$ of the patients) and anxiety disorders (in $21 \%$ ) were the most common diagnoses. Older patients were mainly diagnosed of depressive disorders $(p=0.016)$ whereas younger patients tended to receive diagnoses of anxiety disorders $(p=0.039)$. Conclusion: Patients presenting with FSS may have diagnosable psychiatric disorders which if not recognized would go untreated. Psychiatric referral may therefore reduce the sufferings of the patients.
\end{abstract}

${ }^{*}$ Corresponding author.

How to cite this paper: Okafor, C. J., Udofia, O., \& Ekpe, E. E. (2015). Psychiatric Diagnoses of Patients Presenting with Functional Somatic Symptoms in a Primary Care Clinic at a University Teaching Hospital in Nigeria. Open Journal of Depression, 4, 31-40. http://dx.doi.org/10.4236/ojd.2015.43004 
Keywords

Somatization, Functional Somatic Symptoms, Depression, Anxiety

\section{Introduction}

A significant number of patients seen in primary care clinics complain of physical symptoms which are not attributable to any known conventionally defined physical disorder. These are sometimes said to be medically unexplained or Functional Somatic Symptoms (Fink, Rosendale, \& Olesen, 2005). These Functional Somatic Symptoms (FSS) have generated so much interest among psychiatrists working among Africans in Africa or in the Diaspora. Some of these symptoms such as the sensation of internal heat, peppery feeling and crawling sensations are so common amongst depressed Africans that some authors have gone on to state that depressed Africans do not manifest the traditional symptoms of depression (Ihezue, 1986). It has also been stated that African patients with psychiatric disorders present with Functional Somatic Symptoms, a process which has been referred to as somatization (Ebigbo \& Ihezue, 1982; Morakinyo, 1985; Okulate, Olayinka, \& Jones, 2004).

At present, the diagnoses of patients with functional somatic symptoms remain controversial. The current situation is that while contemporary psychiatry subsumed functional somatic complaints under the diagnostic category of somatoform disorders, some clinicians in Africa have observed that the same somatic complaints could attract various psychiatric diagnoses (Ebigbo \& Ihezue, 1982). Okulate et al. observed that somatic complaints have little weight in the diagnosis of depression but opined that they may have more weight in the diagnosis of anxiety disorders. It is therefore yet to be understood whether somatization is a psychiatric disorder as presently conceptualized under somatoform disorder in the current classification system or whether the condition is a manifestation of anxiety, depression or other psychiatric disorders. It is important to resolve this issue since psychiatric disorders like depression and anxiety can be satisfactorily treated if diagnosed early.

The above controversy notwithstanding, there seem to be an agreement amongst clinicians that somatizing patients are difficult to treat (Ohaeri \& Olatawura, 1988; Ebigbo, Janakiramaiah, \& Kumaraswamy, 1986). Ohaeri and Olatawura drew attention to the resilience of somatic complaints to physical method of treatment. The difficulties encountered in treating somatizing patients might stem from our limited understanding of the condition. To better our understanding of somatizations, we conducted this study which major aim was to determine the International Classification of Diseases-10 $0^{\text {th }}$ edition (ICD-10) psychiatric diagnoses (using standardized diagnostic instrument) in patients that presented with functional somatic complaints. We believe that if the psychiatric diagnoses in patients presenting with functional somatic complaints are well understood, it will help in facilitating an effective treatment for this condition.

\section{Methods}

\subsection{Location of Study}

The study was carried out in General Out-Patients Clinics (GOPC) of the University of Calabar Teaching Hospital (UCTH). Although a tertiary hospital, the UCTH also serves as the main primary health care centre for Calabar metropolis, taking both walk-in and referred patients. Consultant family physicians and resident doctors in family medicine run the daily outpatient clinics in the hospital. Clinics are conducted daily with the exception of Saturdays and Sundays. An average of six hundred and fifty (650) patients attends the clinic every week. Patients for this study were recruited from the GOPC of the UCTH.

\subsection{Ethical Consideration}

Permission to carry out this study was obtained from the ethical committee of hospital. The aims and objectives of the study were explained to each patient and only those who gave their consent participated in the study.

\subsection{Population Sample}

The population studied consisted of adult patients (18 years and above) seeking non-emergency care at the 
GOPC of the UCTH between Monday the 2nd of March and Friday the 22nd of May 2009. The criterion for including a patient in the study was presenting in the hospital with any one or combination of somatic symptoms provided that the symptoms were not attributable to organic pathology by the family physicians. The exclusion criteria included: inability to communicate in English language; patients taking medications known to cause psychiatric symptoms (such as alpha methyl dopa, corticosteroids and cimetidine); those whose complaints were attributed to organic pathology and those that had previous contact with psychiatric services.

\subsection{Instruments}

Two instruments were used for this study. These instruments were:

1) Modified version of the Enugu Somatization Scale with few more somatic questions from the work of Morakinyo (Morakinyo, 1985). The Enugu Somatization Scale (Ebigbo, 1981) consists of 65 somatic complaints compiled exactly as reported by Nigerian psychiatric patients. We considered 21 of these 65 complaints not specific enough and as such excluded them leaving us with only 44 items. The excluded complaints include "I know that my body is not alright", "it is difficult to explain what is wrong with my body", "I am convinced that only traditional healers can do the jobs on me" amongst others. We added six more somatic questions from the works of Morakinyo to the 44 remaining items in the ESS. The resultant hybrid questionnaire consists of 50 items (this is the modified Enugu Somatization Scale). This questionnaire served as check list to aid the patient remember the somatic symptoms (s)he has.

2) Schedule for Clinical Assessment in Neuropsychiatry (SCAN) (Wing, Babor, Brugha et al., 1990). Each of the 100 patients with unexplained somatic symptoms also went through a psychiatric interview assisted with the SCAN. The SCAN is a set of semi-structured instruments aimed at assessing, measuring and classifying the psychopathology and behavior associated with the major psychiatric syndromes. It was developed by the World Health Organization (WHO) and the National Institute for Health (NIH) joint project on diagnoses. Its components include: the Present State Examination-10 ${ }^{\text {th }}$ edition (PSE-10), the Glossary of Differential Definitions, Item Group Checklist (IGC) and the Clinical History Schedule (CHS). The SCAN systems also contain another essential element called the CATEGO which is a set of computer program for processing SCAN data and providing output. With the SCAN, diagnosis can be made on any of the following states of the patient: Present state, Lifetime before and a Representative previous episode. The use of SCAN requires training which two persons in this research team received in a WHO accredited centre. These trained researchers deployed the computerized version of the SCAN to make the present state diagnoses (ICD-10 categories) of the 100 randomly selected subjects that participated in the study.

\subsection{Pilot Study}

To determine the reliability of the modified Enugu Somatization Scale (modified ESS) and ascertain the feasibility of this study, a pilot study was conducted where the family physicians were asked to identify and refer (to the research team) patients who presented to them with somatic complaints which they considered were not attributable to organic pathology. A total of nine patients were referred and each was given the modified ESS to complete. SCAN was also administered on each of these patients and their mobile phone numbers and/or contact addresses collected. These patients were again seen after an interval of one week and the questionnaire (modified ESS) was again completed by each of them. At the end of the exercise, a reliability coefficient of 0.933 was obtained for the modified ESS. The pilot study also revealed that it took an average of 2 hours to complete a SCAN interview. For this reason, the number of patients to be interviewed with the SCAN was pegged at 4 for each clinic day.

\subsection{Procedure}

In order to interview (with the SCAN) a maximum of four (4) patients per clinic day, a number from 1 to 4 was randomly selected and assigned to each of the consulting doctor every clinic day. This was to enable each doctor identify a particular patient to be referred amongst those that presented to him/her with somatic complaints in the sequence of their consultations. For example, a doctor that was assigned number 3 - on a clinic day was required to identify and refer the third patient amongst those that presented to him with somatic complaints after excluding organic causes. Similarly, the doctor that was assigned number 1 identified and referred the first pa- 
tient that presented to him with such somatic complaints. After consultations with the physicians, every patient that was selected and referred by the physicians was recruited into this study provided that he/she consented to participate. The selected subjects completed the questionnaires used in this study and were thereafter interviewed using the computerized version of the SCAN. This procedure was followed every Monday to Friday until 100 patients were interviewed with the SCAN.

\subsection{Data Management}

Data from SCAN interview were collected based on the patient's present state and computer algorithms were run to generate the possible psychiatric diagnoses (ICD-10 categories). Data obtained with Socio-demographic questionnaire were subjected to descriptive statistical analysis using Statistical Package for Social Science version 11. Chi-square test was applied to test the difference between variables of categorical data and significance level used was 0.05 .

\section{Results}

A total of 100 patients whose somatic complaints were not attributed to organic pathology by the family physicians were randomly selected and recruited into this study.

\subsection{Demographic Characteristics}

Table 1 shows the demographic characteristics of the 100 patients. Their age range was $20-78$ years with mean age of 38.5 and a standard deviation of 12.2 .

Table 1. Demographic characteristics of the 100 selected patients.

\begin{tabular}{|c|c|c|}
\hline Variable & Frequency & Percent (\%) \\
\hline \multicolumn{3}{|l|}{ Gender } \\
\hline Male & 41 & $41 \%$ \\
\hline Female & 59 & $59 \%$ \\
\hline \multicolumn{3}{|l|}{ Age (years) } \\
\hline$<30$ & 17 & $17 \%$ \\
\hline $30-49$ & 32 & $32 \%$ \\
\hline $50-69$ & 35 & $35 \%$ \\
\hline $70 \&$ above & 16 & $16 \%$ \\
\hline \multicolumn{3}{|l|}{ Marital status } \\
\hline Single & 45 & $45 \%$ \\
\hline Married & 40 & $40 \%$ \\
\hline Separated & 5 & $5 \%$ \\
\hline Widowed & 10 & $10 \%$ \\
\hline \multicolumn{3}{|l|}{ Work status } \\
\hline Student & 19 & $19 \%$ \\
\hline Unemployed & 18 & $18 \%$ \\
\hline Employed & 26 & $26 \%$ \\
\hline Self-employed & 37 & $37 \%$ \\
\hline
\end{tabular}




\subsection{Somatic Symptoms}

The most prevalent somatic symptom ticked by the patients was "frequent headache" and 31 of the patients reported this symptom. Also commonly ticked were "body heat/internal heat" reported by 30 patients and "dizziness" by 28. "Pains all over the body and Palpitations" were also very common. The other common symptoms are shown in Table 2.

\subsection{Psychiatric Diagnoses}

An ICD10 diagnosis was made on $79 \%$ of 100 patients following the SCAN interview. The most prevalent psychiatric disorder diagnosed was depressive disorders. This was diagnosed in 48 patients. As shown in Table 3, Thirty-seven patients (37) had mild depressive disorders and 11 moderate. Anxiety disorders were diagnoses in 21 patients and 10 patients were diagnosed as having somatoform disorders.

Table 4 compares the socio-demographic characteristics of subjects with SCAN diagnoses and that of those

Table 2. Common symptoms reported by the respondents.

\begin{tabular}{|c|c|}
\hline Symptoms & Frequency of respondents \\
\hline Frequent headache & 31 \\
\hline Body heat/internal heat & 30 \\
\hline Dizziness & 28 \\
\hline Pain all over the body & 21 \\
\hline Heart beating fast (palpitations) & 20 \\
\hline Poor appetite & 20 \\
\hline Weight loss & 16 \\
\hline Backache/waist pain & 14 \\
\hline Chest pain & 12 \\
\hline Crawling sensation on the body & 11 \\
\hline Heat in the head & 11 \\
\hline Heaviness in the head & 11 \\
\hline Painful spots in the head & 10 \\
\hline Shakiness of the hands & 9 \\
\hline Weak erection/sexual arousal & 8 \\
\hline Body weakness & 8 \\
\hline Crawling in the head & 7 \\
\hline Excessive sweating without adequate exercise & 7 \\
\hline Shoulder is heavy & 7 \\
\hline Movement in the stomach & 6 \\
\hline Peppery feeling on the body & 6 \\
\hline Pain in the limb(s) & 6 \\
\hline Cant see properly & 6 \\
\hline Lack of balance while walking & 6 \\
\hline Eye pains & 6 \\
\hline
\end{tabular}


Table 3. Distribution of SCAN diagnoses in the subjects.

\begin{tabular}{ccc}
\hline SCAN diagnoses & Frequency & Percent (\%) \\
\hline Depressive disorders & 48 & \\
Mild depression & 37 & $48 \%$ \\
Moderate depression & 11 & \\
\hline Anxiety disorders & 21 & \\
Agoraphobia & 1 & $21 \%$ \\
Generalized anxiety disorder & 14 & \\
Specific phobia & 6 & \\
\hline Somatoform disorders & 10 & $10 \%$ \\
Somatization disorder & 1 & $21 \%$ \\
Undifferentiated somatoform disorder & 9 & $100 \%$ \\
\hline No SCAN diagnosis & 21 & \\
Total & 100 & \\
\hline
\end{tabular}

Table 4. Demographic characteristics of subjects with SCAN diagnoses.

\begin{tabular}{cccc}
\hline \multicolumn{1}{c}{ Variables } & $\begin{array}{c}\text { With SCAN } \\
\text { diagnoses }\end{array}$ & $\begin{array}{c}\text { Without SCAN } \\
\text { diagnoses }\end{array}$ & Statistics \\
\hline Gender & $33(80.5 \%)$ & $8(19.5 \%)$ & $\mathrm{X}^{2}=0.09$ \\
Male & $46(78 \%)$ & $13(22 \%)$ & $\mathrm{Df}=1$ \\
Female & $39(79.6 \%)$ & $10(20.4 \%)$ & $\mathrm{X}=0.76$ \\
Age (years) & $40(78.4 \%)$ & $11(21.6 \%)$ & $p=0.92$ \\
$<50$ & & $7(17.5 \%)$ & $\mathrm{df}=1$ \\
$50 \&$ above & $14(23.3 \%)$ & $p=0.65$ \\
Marital status & $33(82.5 \%)$ & & $\mathrm{X}^{2}=0.20$ \\
Married & $46(76.7 \%)$ & $13(20.6 \%)$ & $\mathrm{df}=1$ \\
Others & & $8(21.6 \%)$ & $p=0.89$ \\
Work status & $50(79.4 \%)$ & & \\
Employed & $29(78.4 \%)$ & & \\
Others & &
\end{tabular}

with no SCAN diagnosis. As can be seen from the table, $80.5 \%$ of the males and $78 \%$ of the females that participated in this study had SCAN diagnoses. The table shows that there is no significant difference in socio-demographic characteristics of these subjects.

Table 5 shows the socio-demographic characteristics of patients diagnosed of depressive disorders. The female to male ratio of those diagnosed of depressive disorder with the SCAN was 1.8:1. The table shows that there was a significant finding in age group of those diagnosed of depressive disorders. Older somatizing patients (50 years and above) were more likely than the younger ones to have a diagnosis of depression $(p=0.016)$. With regards to the occupational status, somatizing patients that were employed were more likely to have a diagnosis of depression $(p=0.003)$.

As shown in Table $6,34.1 \%$ of the entire male and $11.9 \%$ of all the female patients that participated in this study were diagnosed of anxiety disorders. Significant difference was noticed in the gender status indicating that 
Table 5. Demographic characteristics of patients with SCAN diagnosis of depression.

\begin{tabular}{cccc}
\hline \multicolumn{1}{c}{ Variables } & Depression & Other diagnoses & Statistics \\
\hline Gender & & & $\mathrm{X}^{2}=1.19$ \\
Male & $17(41.5 \%)$ & $24(58.5 \%)$ & $\mathrm{df}=1$ \\
Female & $31(52.5 \%)$ & $28(47.5 \%)$ & $p=0.28$ \\
Age (years) & & & $\mathrm{X}^{2}=5.81$ \\
$<50$ & $17(34.7 \%)$ & $32(65.3 \%)$ & $\mathrm{df}=1$ \\
$50 \&$ above & $31(60.8 \%)$ & $20(39.2 \%)$ & $p=0.016^{*}$ \\
Marital status & & & $\mathrm{X}^{2}=0.28$ \\
Married & $21(52.5 \%)$ & $19(47.5 \%)$ & $\mathrm{df}=1$ \\
Others & $27(45.0 \%)$ & $33(55.0 \%)$ & $p=0.597$ \\
Work status & & & $\mathrm{X}^{2}=9.06$ \\
Employed & $38(60.3 \%)$ & $25(39.7 \%)$ & $\mathrm{df}=1$ \\
Others & $10(27.0 \%)$ & $27(73.0 \%)$ & $p=0.003^{*}$ \\
\hline
\end{tabular}

*Denotes significant finding.

Table 6. Demographic characteristics of patients with diagnosis of anxiety disorders.

\begin{tabular}{cccc}
\hline \multicolumn{1}{c}{ Variables } & Anxiety & Other diagnoses & Statistics \\
\hline Gender & & & $\mathrm{X}^{2}=7.2$ \\
Male & $14(34.1 \%)$ & $27(65.9 \%)$ & $\mathrm{df}=1$ \\
Female & $7(11.9 \%)$ & $52(88.1 \%)$ & $p=0.007^{*}$ \\
Age (years) & & & $\mathrm{X}^{2}=4.28$ \\
$<50$ & $15(30.6 \%)$ & $34(69.4 \%)$ & $\mathrm{df}=1$ \\
$50 \&$ above & $6(11.8 \%)$ & $45(88.2 \%)$ & $p=0.039^{*}$ \\
Marital status & $8(20.0 \%)$ & $32(80.0 \%)$ & $\mathrm{X})^{2}=0$ \\
Married & $13(21.7 \%)$ & $47(78.3 \%)$ & $p=1$ \\
Others & & & $\mathrm{X}^{2}=5.79$ \\
Work status & $8(12.7 \%)$ & $55(87.3 \%)$ & $\mathrm{df}=1$ \\
Employed & $13(35.1 \%)$ & $24(64.9 \%)$ & $p=0.016^{*}$ \\
Others & & & \\
\hline
\end{tabular}

*Denotes significant finding.

males who somatize were more likely to get a diagnosis of anxiety $(p=0.007)$. Another significant finding was in the age group where it was found that younger somatizing patients were more likely to have a diagnosis of anxiety $(p=0.039)$. Also, findings in the occupational status showed that somatizing patients who were not employed were significantly diagnosed of anxiety disorders than those that were employed $(p=0.016)$.

More females $(13.6 \%)$ than males $(4.9 \%)$ were diagnosed of somatoform disorders but this finding was not statistically significant $(p=0.16)$. Also, no significant difference was found in other socio-demographic characteristics of patients with diagnosis of somatoform disorders. 


\section{Discussion}

More than two-third of the subjects interviewed with the SCAN had psychiatric diagnoses. This high rate of psychiatric disorders amongst patients with somatic complaints had been reported by some other researchers (Brown, Golding, \& Smith, 1990; Ohaeri \& Odejide, 1994). Morakinyo had observed that the stigma attached to mental illness in Nigeria might lead to tendency for patients to repress their mental symptoms and replace them with somatic ones. He argued that the illness having been perceived as a physical one would then attract sympathy and become more acceptable to the society (Morakinyo, 1985). In the light of this, it is easy to see reason why a high rate of psychiatric morbidity was arrived at following SCAN interview in this study.

In a national ambulatory medical care survey in the United State, it was reported that about two-third of the patients in whom psychiatric disorders were found, presented with somatic complaint (Brown, Golding, \& Smith, 1990). Goldberg and colleague pointed out that many patients with psychiatric disorder seek care for somatic symptoms (Goldberg \& Bridges, 1988).

The most common SCAN diagnoses reached in this study were Depression and Anxiety disorders. This is not surprising in view of the earlier findings that patients with depression and anxiety frequently complained of somatic symptoms (Makanjuola, 1987; Silver, 1987). Makanjuola observed that depression and anxiety disorders were the most common DSM III diagnoses amongst subjects diagnosed of "Ode-Ori" (a syndrome of somatic complaints) by the traditional healers in Yoruba, Nigeria (Makanjuola, 1987). Outside Nigeria, Hamilton and colleagues found that depression and anxiety were diagnosed in $33 \%$ of patients with medically unexplained symptoms who presented in an out patients clinic at a large teaching hospital (Hamilton, Campos, \& Creed, 1999).

The commonest psychiatric diagnoses reached in this study were depressive disorders. This confirms earlier reports that depression is not as rare in Africa as had been thought (Ihezue, 1986). Binitie in a study of depression across cultures showed that somatic symptoms were prominent features amongst the depressed patients from Benin, Nigeria (Binitie, 1975). Inferring from the above, we can say that core symptoms of depression could be elicited from patients presenting with somatic complaints, if careful interview about their emotional state was done.

Amongst the depressed cases identified in this study, mild depression was the more predominant diagnosis. This is in keeping with Ilechukwu's study which showed that somatic symptoms were common amongst patients with mild depression as those with severe depression tended to have more of psychomotor retardation (Ilechukwu, 1991).

This study found significant difference in age interval of somatizing patients with SCAN diagnosis of depression compared with those with other diagnoses. It was found that the rate of depression increased significantly in somatizing patients who were aged 50 years and above. Although the reason for this is not clear, it is possible that the increased rate of physical disabilities in old people contributed to this increased rate of depression.

With regards to patients in whom SCAN diagnoses of anxiety disorders were reached, significant findings were observed in their age interval, gender and work status. It was found that young somatizing patients were more likely to have anxiety disorder than the older somatizing patients. This finding agrees with that of Awaritefe in a study on clinical anxiety in Nigeria (Awaritefe, 1988). In that study, it was found that the younger patients were more vulnerable to clinical anxiety than the elderly ones. The present study also found that somatizing patients who were males as well as the unemployed were more likely to have anxiety disorder. Our attempts to find a possible reason for these findings are yet to yield any result.

\section{Conclusion}

This study has shown that some of the patients that attend the General Out-Patients Clinic at the University of Calabar Teaching Hospital do so as a result of somatic symptoms which are not attributable to organic pathology. Most of these patients with such symptoms might have psychiatric disorders which if not recognized, would go untreated. For this reason, physicians working at this level should look out for psychiatric disorders in patients that present to them with somatic symptoms which are not attributable to organic pathology. Because of the large number of patients to attend to and the busy schedules of these physicians, they may not have enough time to administer a standardized diagnostic instrument. It is therefore recommended that they refer such patients to psychiatric service for proper evaluation and management by a psychiatrist. In places where psychiatrists are not available, drug trial for anxiety and depressive disorders should be encouraged with the presumption that de- 
pressive disorders tend to occur in older somatizing patients (aged 50 years and above) while the younger somatizing patients especially males, are more likely to have anxiety disorders.

It must be stated that our study has its strength and potential limitations. Although the method of random sampling was adhered to in selecting the subjects that participated in this study yet caution should be applied because the sample size of one hundred is not enough to warrant generalization of findings. Also, undiagnosed organic pathologies may have been responsible for some of the symptoms reported by the subjects.

\section{Acknowledgements}

We owe a debt of gratitude to the following persons who contributed in one way or the other to this study; Mrs. Blessing Okafor for her careful perusal and correction of the manuscript. Prof. Olufemi Morakinyo for the provision of relevant materials needed to improve the quality of this study. Prof. Oye Gureje for the training we received in the use of SCAN. Dr. Timilehen Babatunde for his assistance in data collection. The Family Physicians and the Nurses working at the GOPD of the UCTH for their help and cooperation.

\section{Conflicts of Interest}

There are no conflicts of interest for the authors of this research work.

\section{References}

Awaritefe, A. (1988). Clinical Anxiety in Nigeria. Acta Psychiatrica Scandinavica, 77, 729-735. http://dx.doi.org/10.1111/j.1600-0447.1988.tb05195.x

Binitie, A. (1975). A Factor Analytic Study of Depression across Cultures (African and European). British Journal of Psychiatry, 127, 559-563. http://dx.doi.org/10.1192/bjp.127.6.559

Brown, F. W., Golding, M. J., \& Smith, R. G. (1990). Psychiatry Comorbidity in Primary Care Somatization Disorder. Journal of Psychosomatic Medicine, 52, 445-451. http://dx.doi.org/10.1097/00006842-199007000-00006

Ebigbo, P. (1981). The Development of Culture Specific (Nigeria) Screening Scale of Somatic Complaints Indicating Psychiatric Disturbance. Culture Medicine and Psychiatry, 6, 29-43. http://dx.doi.org/10.1007/BF00049469

Ebigbo, P., \& Ihezue, U. (1982). Uncertainty in the Use of Western Diagnostic Illness Categories for Labeling Mental Illnesses in Nigeria. Psychopathologie Africaine, XVIII, 59-74

Ebigbo, P., Janakiramaiah, N., \& Kumaraswamy, N. (1986). Somatization in Cross-Cultural Perspective. In: K. Peltzer, \& P. Ebigbo (Eds), Clinical Psychology in Africa (pp. 233-241). Enugu: Chuka Printing Press.

Fink, P., Rosendal, M., \& Olesen, F. (2005). Classification of Somatization and Functional Somatic Symptoms in Primary Care. Australian and New Zealand Journal of Psychiatry, 39, 772-781. http://dx.doi.org/10.1080/j.1440-1614.2005.01682.x

Goldberg, D. P., \& Bridges, K. (1988). Somatic Presentation of Psychiatric Illness in Primary Care Setting. Journal of Psychosomatic Research, 32, 137-144. http://dx.doi.org/10.1016/0022-3999(88)90048-7

Hamilton, J., Campos, R., \& Creed, F. (1999). Anxiety, Depression and Management of Medically Unexplained Symptoms in Medical Clinics. Journal of Royal College of Physicians London, 30, 18-20.

Ihezue, U. (1986). The Influence of Sociocultural Factors on Symptoms of Depressive Illness. In: K. Peltzer, \& P. Ebigbo (Eds.), Clinical Psychology in Africa (pp. 217-223). Enugu: Chuka Printing Press.

Ilechukwu, S. T. (1991). Psychiatry in Africa: Special Problems and Unique Features. Transcultural Psychiatric Research Review, 28, 169-218. http://dx.doi.org/10.1177/136346159102800301

Makanjuola, R. O. (1987). “Ode-Ori”: A Culture Bound Disorder with Prominent Somatic Features in Yoruba Nigerian Patients. Acta Psychiatric Scandinavica, 75, 231-326. http://dx.doi.org/10.1111/j.1600-0447.1987.tb02781.x

Morakinyo, O. (1985). Phobic States Presenting as Somatic Complaints Syndromes in Nigeria: Sociocultural Factors Associated with Diagnosis and Psychotherapy. Acta Psychiatric Scandinavica, 7, 356-365.

http://dx.doi.org/10.1111/j.1600-0447.1985.tb02535.x

Ohaeri, J., \& Odejide, O. (1994). Somatization Symptoms among Patients Using Primary Health Care Facilities in a Rural Community in Nigeria. American Journal of Psychiatry, 151, 728-731. http://dx.doi.org/10.1176/ajp.151.5.728

Ohaeri, J., \& Olatawura, M. (1988). The Resilience of Somatic Symptoms to Physical Method of Treatment. Psychopathologie Africaine, XXII, 65-78.

Okulate, G., Olayinka, M., \& Jones, O. (2004). Somatic Symptoms in Depression; Evaluation of Their Diagnostic Weight in 
an African Setting. British Journal of Psychiatry, 184, 422-427. http://dx.doi.org/10.1192/bjp.184.5.422

Silver, H. (1987). Physical Complaints Are Parts of the Core Depressive Syndromes: Evidence from a Cross Cultural Study in Israel. Journal of Clinical Psychiatry, 48, 140-142.

Wing, J. K., Babor, T., Brugha, T., Cooper, J. E., Giel, R., Jablenski, A., et al. (1990). SCAN: Schedules for Clinical Assessment in Neuropsychiatry. Archives of General Psychiatry, 47, 589-593.

http://dx.doi.org/10.1001/archpsyc.1990.01810180089012 